Teil 2

Referentialität und Urheberrecht 



\title{
Kleine Münze, große Fragen. Musikalische Schöpfungshöhe aus der Perspektive empirischer Musikforschung
}

\author{
Klaus Frieler", Daniel Müllensiefen
}

\section{Einleitung}

Das deutsche Urheberrecht schützt persönliche geistige Schöpfungen in verschiedenen kreativen Bereichen. Im Fall der Musik gilt der Urheberrechtsschutz auch für relative geringe schöpferische Leistungen, was in der Rechtspraxis unter dem Begriff der Kleinen Münze beschrieben wird ${ }^{1}$. Für Melodien gilt ein besonderer gesetzlicher Schutz $(\$ 24,2)$, wobei im Gesetz offengelassen wird, was genau eine Melodie konstituiert. Wiedererkennbarkeit und Individualität bzw. Originalität werden in der Rechtsprechung oft als Kriterien für die Schutzfähigkeit herangezogen ${ }^{2}$, aber Erkenntnisse der aktuellen empirischen musikpsychologischen Forschung, die wir hier präsentieren, stellen in Frage, ob kurze Melodien, die unter den rigiden stilistischen Vorgaben kommerzieller Musikproduktion generiert werden, in jedem Fall als persönliche geistige Schöpfungen gelten sollten.

In der folgenden Diskussion argumentieren wir, dass der starre Melodienschutz für heutige Popmusik nicht mehr angemessen sein könnte und die musikalische Massenproduktion zusammen mit neuesten technologischen Entwicklungen in den Bereichen Big Data und künstlicher Intelligenz die Bewertung von musikalischer Schöpfungshöhe und musikalischer Urheberrechtsverletzung bei Melodien an einen „Tipping Point“ geführt

* Dr. Klaus Frieler ist freiberuflicher Musikwissenschaftler. Seine Forschungsschwerpunkte sind rechnergestützte Musikforschung als Methode in der Musikpsychologie und Jazzforschung mit Schwerpunkt auf dem kreativen Prozess. Unter anderem ist er als Musikgutachter tätig.

** Prof. Dr. Daniel Müllensiefen ist Musikpsychologe an der Goldsmiths University of London sowie an der Hochschule für Musik, Medien und Theater in Hannover. Seine Forschungsschwerpunkte sind unter anderem neue Ansätze der Musikalitätsforschung und musikalisches Urheberrecht aus kognitionspsychologischer Perspektive.

1 BGH, Urt. v. 3.2.1988 - I ZR 142/86, Ein bisschen Frieden; Bisges, Kleine Münze.

2 BGH, Urt. v. 24.1.1991 - I ZR 72/89, Brown Girl in the Ring; Hanser-Strecker, Plagiat. 
hat, der eine Änderung der traditionellen Rechtspraxis in diesem Bereich nahelegt. Die Argumentationslinie stützt sich auf die Erkenntnis, dass 1. stilistische Einschränkungen in kommerziell erfolgreichen Popmusikstilen die Verwendung nur bestimmter melodischer Patterns zulassen und 2. in der Produktionspraxis Melodien als Rekombination dieser kurzen Patterns entstehen. Der empirische Nachweis dieser Mechanismen wird durch Analysemethoden aus dem Bereich Big Data ermöglicht, mit Hilfe derer sich vorbestehende melodische Elemente in Popsongs systematisch identifizieren lassen.

Dieser Tipping Point macht es notwendig, den starren Melodienschutz durch differenziertere Beurteilungsverfahren zu ersetzen. Diese Verfahren verwenden melodische Schöpfungshöhe und melodischen Schutzfähigkeit als kontinuierliche Parameter und legen zudem nahe, die Messlatte für melodische Urheberrechtsverletzungen deutlich höher anzusetzen als es in der gegenwärtigen Rechtspraxis der Fall ist.

\section{Generierung melodischer Patterns unter stilistischen Einschränkungen}

Westliche kommerzielle Popmusik ist stark kodifiziert ${ }^{3}$. Kodifizierung bedeutet, dass auf allen musikalischen Ebenen Elemente (relativ) unverändert in vielen Werken zu finden sind. Die Kodifizierung ist kein Alleinstellungsmerkmal von Popmusik, sondern findet sich auch in ähnlicher Weise in der Barockmusik oder der sogenannten „Common Practice“ Kunstmusik. Die starke Kodifizierung kommerzieller Popmusik kommt u. a. dadurch zustande, dass der Druck auf Popkomponisten hoch ist, Werke zu erschaffen, die am Markt bestehen können ${ }^{4}$. Allerdings sind valide explizite Regeln für Markttauglichkeit im Allgemeinen auch den Popmusikkomponisten und -produzenten nicht bekannt, sondern es wird vielfach implizit geurteilt und oft auf „musikalische Intuition“ und Erfahrung verwie$\operatorname{sen}^{5}$.

Die intuitive Beurteilung neuer Popkompositionen wird durch kognitions- und wahrnehmungspsychologische Mechanismen realisiert, die für eine schnelle und erfolgreiche Verarbeitung des musikalischen Materials sorgen. $\mathrm{Zu}$ diesen Mechanismen gehören der mere exposure effect ${ }^{6}$, der vor-

3 Kramarz, Hits; Schmidt/Terhag, Songwriting.

4 Vgl. Bennet, Popular songwriting, in: Collins, The act of musical composition; de Laat, Work and Occupations 2015 225ff.; Seabrook, Songmachine.

5 Vgl. Seabrook, Songmachine.

6 Zajonc JPSP 1968, 1. 
hersagt, dass ästhetische Stimuli bei wiederholter Präsentation als angenehmer empfunden und mit besseren Gefallensurteilen bewertet werden. Ein anderer Mechanismus ist die availability heuristic ${ }^{7}$, die erklärt, warum Konzepte und Stimuli, die besonders einfach aus dem Gedächtnis abgerufen werden können, Urteilprozesse beeinflussen können. Die Verwendung von bestehendem Material stellt zudem geringere Ansprüche an die Informationsverarbeitung beim Hörer, da beim Hören auf implizit gelernte Strukturen zurückgegriffen werden kann, die das chunking ${ }^{8}$, d. h. die Aufteilung in kürzere Sinneinheiten, im Arbeitsgedächtnis erleichtern'. Bei der (intuitiven) Beurteilung von Popsongs kommen also universelle psychologische Mechanismen zum Tragen, die alle eine Tendenz aufweisen, vorbestehendes musikalisches Material als angenehm und ästhetisch positiv zu evaluieren. Das gilt für Konsumenten und Produzenten gleichermaßen, da Produzenten natürlich auch Konsumenten sind, und erfolgreich Schaffende durch ihren Marktbestand gezeigt haben, dass sie die ästhetischen Urteile der Hörer antizipieren können ${ }^{10}$.

Die verschiedenen musikalischen Parameter (z. B. Harmonie, Melodie, Rhythmik) werden dabei nicht einzeln bewertet, sondern im Verbund miteinander. Dies trägt assoziativen statistischen Lernprozessen Rechnung, bei denen musikalische Regeln implizit, d. h. unbewusst, beim Musikhören gelernt werden. Diese assoziativ und unbewusst durch das Hören von Popmusik gelernten Regeln stellen stilistische Grenzen dar, die bedeutsam sind, um den Raum der melodischen Möglichkeiten einzuschränken und sicherzustellen, dass eine Melodie als Popmelodie wahrgenommen wird und zum gewählten harmonischen und rhythmischen Material passt. Für die Sensitivität von Hörern, für die musikalisch-statistischen Regeln und Grenzen musikalischer Genres liegen empirische Nachweise vor. So konnten Müllensiefen, Egermann und Burrows ${ }^{11}$ in einem psychologischen Experiment zeigen, dass Versuchspersonen aus Deutschland und Großbritannien die musikalisch-statistischen Regeln von Radiostationsjingles sehr genau im impliziten Gedächtnis gespeichert haben. Im Experiment wurden

7 Tversky/Kahneman Cognitive Psychology 1973, $207 \mathrm{ff}$.

8 Miller Psychological Review 1956, 81ff.

9 Baddeley, Working Memory.

10 Dabei bleibt allerdings die Frage offen, ob ein Popmusikproduzent erfolgreich sein kann, ohne zumindest teilweise den Geschmack seines Publikums zu teilen. Uns ist aber keine empirische Forschung dazu bekannt, so dass dies erst mal unbeantwortet bleiben muss.

11 Müllensiefen et al., Radio Station Singles, in: Bronner/Ringe/Hirt, Audio Branding Yearbook, 53ff. 
Jingles mit leichten melodischen Manipulationen, die eine Verletzung der Regeln darstellten, als nicht zum Genre gehörig identifiziert.

Die intuitive Beurteilungs- und Produktionspraxis neuer popmusikalischer Schöpfungen hat zur Folge, dass bestimmte kompositorische Elemente (z. B. Harmoniefolgen und Melodiefloskeln) sich fast unverändert über einen langen Zeitraum in der Popmusikgeschichte erhalten, was insbesondere für den Teilbereich der charttauglichen Musik zutrifft ${ }^{12}$. Gleichzeitig finden die eigentlichen musikalischen Innovationen eher in den Bereichen Interpretation, Arrangement, Sound und Aufnahmetechnik statt ${ }^{13}$, die jedoch nicht als schützbare Werkteile im Sinne des Urheberrechts gelten, sondern in den Bereich von Leistungsschutzrechten fallen. Insgesamt bedeutet dies, dass bei Popmusikwerken in der Regel im Vergleich mit der Kunstmusik geringe Anforderungen an die kompositorische Originalität und Individualität gestellt werden. Die Rechtspraxis trägt diesem Umstand mit der sogenannten „Kleinen Münze“ Rechnung, mit der die untere Grenze von gerade noch urheberrechtlich schutzfähigen Werken bezeichnet wird. Dabei werden aber harte, überprüfbare Kriterien nicht gegeben, so dass die hinreichende Schöpfungshöhe in jedem Einzelfall einzeln bestimmt werden muss, was durchaus Quelle für Rechtsunsicherheiten ist.

Wie Musiker haben auch musikalische Laien statistische stilspezifische Regeln im impliziten Gedächtnis abgelegt und können mit Hilfe dieser Regeln unter geeigneten Umständen stilkonforme Musik produzieren. Eine geeignete experimentelle Prozedur zur Untersuchung impliziten stilspezifischen Wissens stellt das „Kreativ-Karaoke-Paradigma“ dar. Die von Frieler und Riedemann ${ }^{14}$ mit diesem Paradigma durchgeführten Experimente belegen, dass auch musikalisch nicht speziell vorgebildete Personen in der Lage sind, stilkonforme Popmelodien zu einer vorgegebenen Begleitmusik in kürzester Zeit (20-30 min) zu erfinden. Die zentrale Frage der Experimente von Frieler und Riedemann zielte auf Häufigkeit und Erklärbarkeit unabhängiger Doppelschöpfungen bzw. unbewusste Übernahmen unter Vorgabe typischer Harmoniefolgen, wie sie in der Popmusik oft vorkommen. Als Stimulusmaterial wurde eine kurze Begleitmusik vorproduziert, die auf einer der gängigsten Popakkordfolgen (I-vi-IV-V) beruhte und im Stil westlicher kommerzieller Popmusik instrumentiert und eingespielt wurde. Die Aufgabe der Versuchsteilnehmer mit unterschiedlicher musikalischer Vorbildung - vom kompletten Laien bis hin zum semi-

12 Vgl. Frith et al., Cambridge Companion; Moore, Analyzing pop music.

13 Vgl. Jörger, Plagiat; Moore, Analyzing pop music.

14 Frieler/Riedemann Musicae Scientiae 2011, S. $17 \mathrm{ff}$. 
professionellen Musiker - bestand darin, zur vorgegebenen Begleitmusik eine Melodie zu erfinden und schließlich einzusingen. Die von den Versuchsteilnehmern produzierten Melodien wurden anschließend musikanalytisch ausgewertet und mit den Melodien von fünf bekannten Popsongs, die dieselbe Akkordfolge zur Grundlage haben, phrasenweise auf Ähnlichkeiten verglichen. In der Tat zeigte sich, dass einer der 30 Versuchsteilnehmer seine Melodie (ohne dessen sich bewusst zu sein) aus zwei melodischen Phrasen zusammengesetzt hatte, die sich jeweils fast identisch in den verwendeten Vergleichssongs wiederfinden („Just like a pill“ von Pink und "What a wonderful world“ von Sam Cooke). Auch bei einer Replikation des Experimentes im Zuge einer Public-Science-Veranstaltung wurde von einer Teilnehmerin ohne formale musikalische Vorbildung „What a wonderful world" fast in Originalgestalt reproduziert. Die Tatsache, dass sich unbewusste Übernahmen von großen Melodienteilen selbst bei Laborexperimenten mit wenigen Versuchsteilnehmern und einer kleinen Zahl an Vergleichsmelodien beobachten lassen, sind ein klarer Hinweis dafür, dass die Verwendung von vorbestehendem melodischen Material in der Praxis häufig vorkommt. Zwar ist es schwierig, die Ergebnisse von Stichproben, die vor allem musikalische Laien, Amateure und Semi-Profis umfassten, auf die Praxis unter professionellen Musikern zu generalisieren, da bei Profimusikern das musikalische Gedächtnis in der Regel noch umfassender und genauer ausgeprägt ist ${ }^{15}$. Es ist aber denkbar, dass die Verwendung von vorbestehendem Material unter Profimusikern mindestens ebenso wahrscheinlich, wenn nicht sogar wahrscheinlicher ist. Allerdings können in professionellen Kontexten, gerade beim modernen Songwriting in Teams Kontrollmechanismen greifen, die unbewusste Übernahmen vorbestehenden Materials abfangen, indem etwa jemand, der an der Produktion des Songs beteiligt ist, auf urheberrechtlich relevante Übereinstimmungen mit bereits veröffentlichtem musikalischen Material hinweist. Allerdings greift dieser Mechanismus nicht immer, wie etwa der prominente Fall von George Harrisons Song „Sweet Lord“ zeigt, bei dem bedeutsame melodische Teile sich im vorbestehenden Song „He‘s So Fine" der Chiffons finden lassen.

15 Halpern \& Bartlett, Memory, in: Riess Jones, Fay, Popper, Music perception, 234ff. 


\subsection{Melodien als Rekombination vorbestehender melodischer Patterns}

Es ist möglich, Melodien in der Popmusik, wie auch in anderen Stilen, als Sequenz von kurzen melodischen Patterns (d. h. Motiven, melodischen Wendungen) zu verstehen und analytisch zu untersuchen. Wenn man sich z. B. die Töne einer Melodie als Folge von Symbolen vorstellt, dann kann man diese Folge in kurze, sich überlappende „Schnipsel“ einer bestimmten Länge aufteilen, die man dann weiter untersuchen kann. Hat man etwa eine Melodie mit den Tönen C-D-E-F-G, dann kann man dies in drei Schnipsel der Länge drei aufteilen, d. h. C-D-E, D-E-F, E-F-G. Anstatt der Tonhöhen kann man aber auch abgeleitete Darstellungsformen untersuchen, z. B. Notenlängen oder Intervalle, d. h. den Unterschied zwischen zwei Tönen. Im vorherigen Beispiel wäre die Intervallfolge $+2+2+1+2$, die man in zwei drei-elementige Schnipsel zerlegen kann: $+2+2+1$ und $+2+1$ +2 . Da Intervalle Unterschiede sind, sind Intervallsequenzen immer ein Element kürzer als die originalen Tonhöhenfolgen, da man für das letzte Element keinen Unterschied mehr angeben kann.

Betrachtet man beispielsweise Jazzimprovisationen, so kann man dort die Produktion von langen Melodielinien in Echtzeit beobachten, deren Komplexität die von Popsongs in der Regel um einiges übersteigt. Aus der Jazzforschung und -pädagogik ist bekannt, dass Jazzmusiker sich beim Improvisieren - zumindest teilweise - vorgefertigter Bausteine bedienen, die Patterns oder Licks genannt werden. So bestehen die Soli von Charlie Parker ausschließlich aus geschickt zusammengesetzten und vorbereiteten melodischen Bausteinen ${ }^{16}$. Analog dazu belegen Analysen eines Corpus von 450 Volksliedern ebenfalls, dass alle Lieder des Corpus komplett aus kurzen Patterns melodischer Intervalle bestehen, die häufig vorkommen ${ }^{17}$. So konnte für diesen Corpus von 50 irischen, 200 polnischen Volkslieder sowie 200 deutschen Kinderlieder nachgewiesen werden, dass diese zu $100 \%$ aus viertönigen Intervallmustern bestehen, die in mindestens drei verschiedenen Melodien vorkommen. Das heißt, dass keines der Volkslieder der Korpora auch nur ein einziges viertöniges Intervallmuster beinhaltet, das nicht auch in mindestens zwei weiteren Liedern zu finden ist.

16 Frieler, Patternology, https://medias.ircam.fr/x54b408 (zuletzt abgerufen am 15.07.2020); Norgaard Music Perception 2014, 271ff.; Owens, Charlie Parker.

17 Frieler, Rhythmen- und Tonhöhenmuster. 


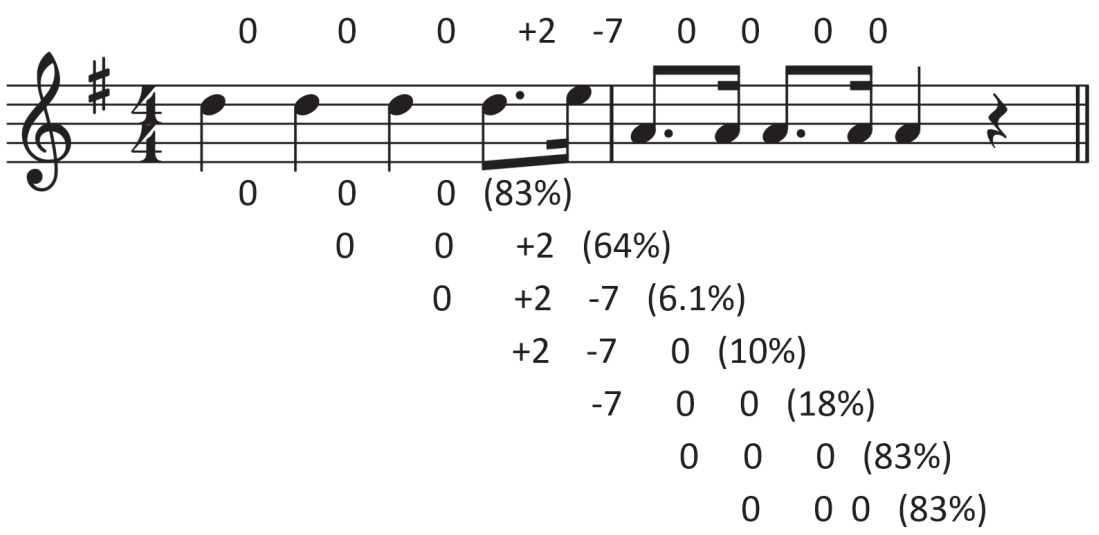

Abbildung 1. Anfang des Refrains von „Yellow Submarine“ (The Beatles). Oberhalb der Noten: Abfolge der Halbtonintervallen. Unterhalb der Noten: Die aufeinanderfolgenden Intervall-3-gramme. In Klammern der Anteil der Songmelodien im Popkorpus, die dieses 3-gramm enthalten.

Ähnliches gilt auch für Popmusikmelodien. Um dies zu zeigen, haben wir eine Sammlung von 12.000 Popsongmelodien ${ }^{18}$ in Hinsicht auf deren melodischen Bestandteile analysiert. Dazu haben wir alle Melodien mit Hilfe eines Computerprogramms in kurze Intervallfolgen („n-grams“) von 3 bis 6 Elementen ( 4 bis 7 Tonereignissen) zerlegt und analysiert, welcher Anteil einer Melodie aus melodischen Bausteinen („Patterns“) besteht, die auch in weiteren Songs des Korpus vorkommen. Dabei kann man verschiedene Kriterien an die Bausteine stellen, z. B. wie oft diese in der gesamten Sammlung vorkommen oder in wie vielen verschiedenen Melodien. In Abb. 1 kann man ein Beispiel sehen. Dort ist eine kurze Folge von 10 Tönen zuerst in eine 9-elementige Intervallfolge übersetzt worden (oberhalb der Noten). Daraus wurde dann eine Folge von 3-grammen abgeleitet, deren Anfangspunkt sich jeweils um ein Element nach rechts verschiebt. Für jedes Intervall-3-gramm ist dort der Anteil der Songs im Korpus angegeben, die dieses 3-gramm enthalten, z. B. kommt die dreifache Tonwieder-

18 s. Beschreibung dieses Korpus kommerzieller westlicher Popmusik in Müllensiefen et al., High-Level Feature Descriptors, in: Schneider, Hamburger Jahrbuch, $133 \mathrm{ff}$. 
holung 000 , die in der Tonfolge selbst dreimal auftaucht, in $83 \%$ aller Songs vor.

Die Tonfolgen haben wir zum einen nur durch die Intervalle, wie im Beispiel, zum anderen durch eine Kombination von Intervallen, zeitlichen Abstand (interonset-interval, IOI) und metrischer Startposition dargestellt. Für die Intervalldarstellung der Melodien ergibt sich, dass eine typische Popsongmelodie komplett aus Folgen von 5 Tönen (d. h. vier Intervallen) bestehen, die in mindestens 100 weiteren Songs des Corpus vorkommen. Das erklärt sich vor allem aus der hohen Häufigkeit von Tonwiederholungen: im Schnitt sind $25 \%$ einer Popsongmelodie rhythmisierte Tonwiederholungen. Dies lässt sich u. a. dadurch erklären, dass viele Teile von Popsongmelodien ihren Ursprung in rhythmisierter Sprache haben, vor allem in den Strophen der Songs. Der hohe Anteil an melodischen Tonwiederholungen lässt die rhythmische und metrische Dimension der Gesangslinien hervortreten, was sich auch am hohen Anteil von Synkopen (Betonung von eigentlich „unbetonten“ Zeitpunkten im Takt) zeigt. Der Synkopenanteil liegt bei den Melodien des Popkorpus ebenfalls bei 25-30\%.

Die Repräsentation von Popmelodien als kombinierte Sequenzen aus Tonhöhen, Tondauern und metrischer Startposition hat den Vorteil, dass verschiedene Rhythmen bei identischer Tonhöhenfolge als unterschiedliche melodische Bausteine behandelt werden. Verwendet man diese kombinierte Repräsentationsform, zeigen Popsongmelodien eine wesentlich geringere Redundanz in Hinsicht auf die Verwendung melodischer Bausteine. Im Mittel kann man $46 \%$ eines Popsongs mit Viertonmustern abdecken, die in mindestens 50 verschiedenen Melodien vorkommen. Senkt man die Anforderungen an die Häufigkeiten, so findet sich eine Abdeckung mit $91 \%$ (bzw. $71 \%$ oder $57 \%$ ) durch viertönige Bausteine, die in mindestens 2 (bzw. 10 oder 25) verschiedenen Melodien vorkommen. Sechstönige Bausteine, die in mindestens 2 (bzw. 10 oder 25) Popsong vorkommen, machen im Schnitt immer noch $40 \%$ (bzw. 12\% oder $7 \%$ ) einer Melodie aus.

Wie viel spezifisches Material Popmelodien enthalten, $d$. h. wie groß der Anteil von melodischen Bausteinen ist, den sich Popsongs nicht mit anderen Popsongs teilen, die also spezifisch für eine Songmelodie sind, ist grafisch in Abb. 2 dargestellt. In der kombinierten Repräsentationsform (linke Seite der Grafik) sieht man, dass im Schnitt weniger als 10\% der verwendeten melodischen Bausteine der Länge 3 (d. h. 3 Intervalle, also 4 Töne) einer Popmelodie nur in diesem einem Song vorkommen. In der Tendenz bestehen also mehr als $90 \%$ einer Popmelodie aus melodischem Material, das auch in anderen Songs vorkommt. Wie die linke Seite von Abb. 2 zeigt, nimmt der Anteil von originären melodischen Bausteinen 
mit der betrachteten Bausteinlänge deutlich zu und liegt bei Länge 6 (d. h. 7 Töne) bei etwa $85 \%$.

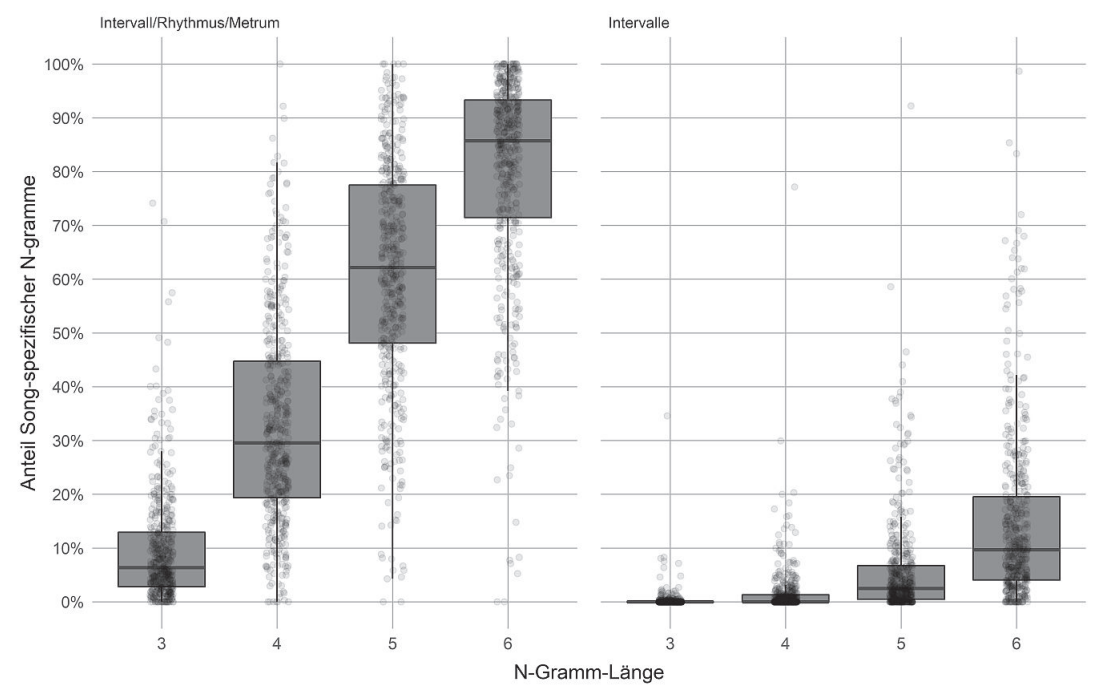

Abbildung 2. Anteil von kurzen Melodiebausteinen, die spezifisch für eine Popsongmelodie ist (aufgeschlüsselt nach Darstellungsform und Länge der Bausteine). Links: Bezogen auf eine Darstellung von Bausteinen mit einer Kombination von Intervallen, Dauern und metrischen Startpositionen; rechts: als Tonhöhenintervalle. Man beachte, dass es sich hier um Differenzdarstellungen handelt, $\mathrm{d}$. h. ein melodischer Baustein mit N Intervallen entspricht N-1 Tönen (Darstellung einer Stichprobe von 1246 Songs).

Betrachtet man allerdings Bausteine in der Tonhöhenintervalldarstellungsform (rechte Seite der Abbildung), dann ist der Anteil der originären Bausteine weitaus geringer. Selbst bei einer Bausteinlänge von 6 besteht ein Popsong im Schnitt noch aus $90 \%$ Material, dass sich auch in anderen Popsongs findet. Auch dies ist der hohen Prävalenz von Tonwiederholungen und kleinen Intervallen geschuldet.

Zusammenfassend zeigt diese kleine Korpusanalyse erstens, dass die Bewertung von melodischen Ähnlichkeiten zwischen Popsongs sich auf eine mehrdimensionale Repräsentationsform stützen sollten, die nicht alleine Informationen zum Tonhöhenverlauf, sondern auch rhythmische und metrische Informationen enthält. Andernfalls ist es kaum möglich, spezifische 
Charakteristika von Popmelodien zu identifizieren, die nicht auch in vielen anderen Popmelodien vorkommen. Dies zeigt überdies die allgemein sehr große Bedeutung der rhythmischen Komponente von Popsongmelodien.

Zweitens legt die Korpusanalyse offen, dass Popmelodien in der Tendenz erst dann wirklich individuelle melodische Bausteine enthalten, wenn man melodisch-rhythmisch-metrische Sequenzen von 5 und mehr Tönen betrachtet. Für die Übernahme kürzerer Motive gilt, dass diese mit großer Wahrscheinlichkeit auch in vielen anderen Songs auftauchen und somit als gemeinfrei gelten können.

Die Verwendung großer Musikdatenbanken und entsprechender Mustererkennungs-Algorithmen findet sich mittlerweile auch in konkreten Urheberrechtsfällen, wie z. B. im Streit um den Katy Perry Song „Dark Horse“. Ein von 15 internationalen Musikwissenschaftlern gezeichneter Amicus Curiae Brief ${ }^{19}$ fordert die Verwendung objektiver Vergleichsmethoden für melodischen Übernahmen ein. Im konkreten Fall wird die große Zahl vorbestehender Werke angeführt, die den streitgegenständlichen melodischen Baustein ebenfalls enthalten. Die vorbestehenden Werke wurden mithilfe von frei zuganglichen Melodiendatenbanken (themefinder.org für Volkslieder und klassische Themen und die RISM Datenbank für musikalische Inzipits klassischer und vorklassischer Themen) identifiziert.

Das Prinzip der Rekombination von bekannten Bausteinen ist nicht auf Melodien beschränkt, sondern lässt sich in allen musikalischen Dimensionen finden. So ist wohlbekannt, dass etwa bestimmte Akkordfolgen immer wieder in der Popmusik verwendet werden ${ }^{20}$. Dasselbe kann man für andere musikalische Einzeldimensionen (z. B. rhythmische Begleitfiguren, Basslinien, Drumgrooves etc.) feststellen, auch wenn für diese Dimensionen detaillierte empirische Korpusstudien noch fehlen. Elemente nichtmelodischer musikalischer Dimensionen gelten aber in der Regel als Gemeingut und ihre Verwendung ist somit keine persönliche geistige Schöpfung, die Urheberrechtsschutz genießt, sondern wird als Ergebnis reinen

19 Freundlich, Brief of Amicus Curiae, https://www.digitalmusicnews.com/wp-conte nt/uploads/2020/01/Katy_Perry_Flame_514_Amici_Brief_Filed.pdf (zuletzt abgerufen am 15.07.2020).

20 Axis of Awesome, 4 Chords, https://www.youtube.com/watch?v=oOlDewpCfZQ (zuletzt abgerufen am 15.07.2020); Frieler/Müllensiefen, Song and Harmonic Templates, in: Tillesen/Prill, Whatever. 
musikalischen Handwerks angesehen ${ }^{21}$. In Angesicht der obigen Erkenntnisse in Bezug auf die Entstehung von Popsongmelodien müsste man dann a fortiori ähnliches auch für Melodien annehmen, d. h. Melodiekomposition ist demnach ebenfalls als musikalisches Handwerk anzusehen. Die im Grunde industriellen Produktionsverfahren im modernen Popmusikbetrieb $^{22}$ sind ein weiteres Indiz dafür, dass es sich dabei nicht um persönliche Schöpfungen sui generis handelt, sondern um quasi-industriell hergestellte Massenprodukte (was aber wohlgemerkt nichts über deren ästhetischen Qualität aussagt).

\subsection{Melodiekomposition als musikalisches Handwerk}

Die vorangegangenen empirischen Ergebnisse zeigen, dass die schöpferische Leistung bei der Komposition von Popmelodien vor allem in der $\mathrm{Zu}$ sammenstellung von bekanntem Material besteht, das aber selbst nicht schützenswert ist. Dies ist keine prinzipiell neue Erkenntnis, denn Melodien bestehen aus Einzeltönen (und deren zeitliche Relation), die für sich genommen nicht schützenswert sind. Die angeführten Belege zeigen aber, dass das Prinzip der Rekombination von vorbestehendem und noch schutzfähigen Material auch auf der nächsthöheren Ebene gilt; der Ebene von kurzen Tonfolgen. Allerdings gibt es einen entscheidenden Unterschied. Kurze Tonphrasen können eigenständige Werkteile im Sinne des Urheberrechts und der Kleinen Münze sein. Es lässt sich eben nicht ausschließen, dass eine kurze melodische Phrase neues originäres Material im Kontext von Popmusik darstellt, trotz der oben angeführten statistischen, psychologischen und produktionstechnischen Argumente, dass die meisten Popsongs vorrangig bekannte melodische Bausteine verwenden.

Während der Einzelton in der Tat vollkommen neutrales und gemeinfreies Material ist, kann man dies für kurze Tonfolgen nicht mehr in voller

21 Allerdings kann die Kombination mehrerer nicht schützenswerter Elemente im Ergebnis zu einem schutzfähigen musikalischen Objekt führen. So stellte etwa das Landgericht München im Fall Still got the Blues fest, dass die einzelnen streitgegenständlichen Dimensionen, d. h. Melodik, Rhythmik, Harmonik, Sound, nur Elemente enthalten, die für sich genommen zum musikalischen Allgemeingut gerechnet werden müssen, dass aber die spezifische Kombination dieser Elemente den musikalischen Gesamteindruck beeinflusst und urheberrechtlichen Schutz genießt. LG München, Urt. v. 3.12.2008 - 21 O 23120/00, Still got the Blues; vgl. auch Döhl, Substantially Similar, in: Bung/Gruber/Kühn, Plagiate.

22 Seabrook, Songmachine. 
Allgemeinheit aufrechterhalten. Die Frage ist dann, wo man sinnvoll Grenzen ansetzen kann, die die Gemeinfreiheit des musikalischen Materials von der Schutzfähigkeit eines originären Werkteils abgrenzen. Dies können aber, wie man leicht feststellt, keine absoluten Grenzen sein. Denn man kann zu jeder Tonfolge einen weiteren Ton hinzufügen, was nur eine geringfügige Änderung bedeute, also einen graduellen Wandel. Dann hat man aber das Paradox, dass eine Menge für sich genommen nicht schutzwürdiger Änderungen an nicht geschütztem Material auf einmal am Ende eine schutzwürdige Schöpfung erzeugt, ohne dass man eine feste Schwelle angeben kann, wann dieser Umschlag von Material zur geistigen Schöpfung passiert ${ }^{23}$.

Aus diesem Grund ist es nicht möglich, melodische Bausteine formal eindeutig zu identifizieren und gegeneinander abzugrenzen. Dies macht es nötig, Bausteine in variable Längen zu betrachten, wie in Abb. 2 geschehen. Zudem gibt es gewisse triviale Veränderungen (z. B. die Aufspaltung einer Viertelnote in zwei Achtelnote derselben Tonhöhe, um z. B. einer Textanpassung vorzunehmen), die perzeptiv nichts an der Identität einer Melodie ändern, und damit in der gängigen Praxis (von Musikgutachtern) als gegenstandlos angesehen werden. Gleichzeitig erschweren diese trivialen Veränderungen die formale Identifizierung von melodischen Bausteinen, und es gilt auch hier, dass eine größere Menge von an sich trivialen Änderungen irgendwann in eine andere Melodie umschlagen kann.

Die einzigen natürliche Einheiten, in die sich Melodien segmentieren lassen, sind perzeptueller Natur und folgen den Prinzipien der auditory scene analysis ${ }^{24}$. So führen z. B. lange Pausen, sehr lange Tondauern oder der Wechsel in eine andere Oktave dazu, dass eine melodische Linie in Phrasen aufgeteilt erscheint. Eine typische Popsongphrase besteht aus 8-10 Tönen, was in etwa zwei viertönigen melodischen Bausteinen entspricht, die als Motiv eine eigene wiedererkennbare melodische Gestalt haben (können). Ausgehend von den Ergebnissen der obigen Korpusanalyse kommen

23 Die einzige logisch ausgezeichnete Schwelle ist die von einem Ton zu zwei Tönen, denn es braucht mindestens zwei Töne, um eine intervallische und rhythmische Relation zu definieren, die ja letztlich das Wesen einer Melodie ausmachen. Allerdings hat die Korpusanalyse ergeben, dass man auch bei Verwendung einer kombinierten Darstellungsform mehr als nur zwei Töne betrachten muss, um originäre melodische Muster in Popmusik identifizieren zu können. Insofern ist die Grenze zwischen einem und zwei Tönen für die Bewertung von Popmelodien kein brauchbares Kriterium.

24 Bregman, Auditory Scene Analysis. 
diese melodischen Bausteine nicht nur in einer einzigen Popmelodie vor, sondern lassen sich auch in vielen anderen Melodien finden (vgl. Abb. 1).

Angesichts dieser empirischen Ergebnisse lässt sich der starre Melodienschutz des deutschen Urheberrechts wie auch die Praxis der Kleinen Münze kaum rechtfertigen. Zumindest muss die Maßgabe, dass auch die Übernahme von (Melodie-)Teilen aus anderen Werken nicht gestattet ist, fallen gelassen oder modifiziert werden, da kurze Teile von Melodien (Phrasen, Motive) als allgemein verfügbarer Bausteine angesehen werden müssen. Einzig längere Konfigurationen von Motiven und Phrasen könnten überhaupt als originäre, d. h. hinreichend differenzierte Rekombinationen von Bausteinen dann noch in Frage kommen ${ }^{25}$.

\subsection{Konsequenzen}

Die vorangegangene Argumentation impliziert eine Reihe von Konsequenzen für die Bewertung von melodischen Übereinstimmungen in der Rechtspraxis. Es sollte offensichtlich sein, dass etwaige Urheberrechtsverletzungen bei Melodien umfassender bewertet werden müssen als dies gegenwärtig der Fall ist, wo oft der Vergleich von kurzen Sequenzen von Tonhöhenfolgen im Fokus steht ${ }^{26}$. Zusätzlich braucht die Identifizierung von Übereinstimmungen und die Ermittlung möglicher Vorbekanntheit melodischen Materials strengere und objektiv nachvollziehbare Maßstäbe. Dabei kann der Einsatz spezialisierter Software ${ }^{27}$ und großer Musikdatenbanken (z. B. die Weimar Jazz DB, themefinder.org) helfen. Die eingesetzte Technologie muss aber auf (musik-)psychologischen Grundlagen stehen, wie z. B. auf Tverskys Theorie zur Ähnlichkeitswahrnehmung ${ }^{28}$, oder über das statistische Lernen von musikalischen Sequenzen ${ }^{29}$. Dazu bedarf es aber auch menschlicher Gutachter mit hinreichendem technischem und psychologischem Sachverstand, um musikalische Korpora und die dazuge-

25 Allerdings argumentiert Fishman (2018), dass die klaren gesetzlichen und rechtspraktischen Vorgaben in Hinsicht auf Melodie zumindest für etwas Rechtssicherheit bei den Produzenten sorgen. Ein Urheberecht, das auf komplexe Kombinationen und den Gesamteindruck abgestellt ist, wäre vielleicht dem Sachverhalt angemessener, aber würde mögliche Rechtsverletzungen noch schwieriger abschätzbar machen.

26 Döhl, Substantially Similar, in: Bung/Gruber/Kühn, Plagiate.

27 Z.B. Cason/Müllensiefen International Review of Law, Computer \& Technology 2012, 25ff.; Müllensiefen/Frieler Computing in Musicology 2004, o.S.

28 Müllensiefen/Pendzich Music Scientiae 2009, $257 \mathrm{ff}$.

29 Pearce/Wiggins Music Perception 2006, $377 \mathrm{ff}$. 
hörigen Algorithmen sinnvoll für urheberrechtliche Fragen einsetzen zu können. In einem kürzlich erfolgten Urteil in dem prominenten Urheberechtsfall „You Raise Me Up“ (Josh Groban) vs. „Into the Light“ (Jóhann Helgason) zeigt sich eine interessante neue Entwicklung in diese Richtung $^{30}$. Dort ließ der Richter André Birotte Jr. das Gutachten für die Klageseite der Musikwissenschaften Judith Finell als „unreliable, unhelpful, and inadmissible" nicht zu, da ihm keine zuverlässige Methodologie zugrunde liege. Dieser Vorwurf wird vor allem damit begründet, dass das Gutachten von Finell gemeinsame Ähnlichkeiten der beiden streitgegenständlichen Songs zum gemeinfreien Song „Londonderry Air“ („Danny Boy“) nicht berücksichtigt hatte - im Gegensatz zum Gutachten der Gegenseite von Lawrence Ferrara. Dies führte dann zur Abweisung der Klage.

Zusätzlich zu diesen Änderungen in der praktischen Beurteilung von musikalischen Urheberrechtsverletzungen können die hier präsentierten Ergebnisse aber auch Implikationen für das musikalische Urheberrecht selbst haben. Aus der Tatsache, dass Popsongmelodien aus kleinen wohlbekannten melodischen Bausteinen bestehen, könnte gefolgert werden, dass die Kleine Münze keine adäquate Praxis darstellt, da sie die nötige Schöpfungshöhe zu niedrig ansetzt. Das bedeutet, dass Schutz nur für größere melodische Komplexe, z. B. ganze Strophen und Refrains mit mehreren Phrasen und Motiven gelten sollte, nicht aber für die Bestandteile (und Varianten davon). Diese Bestandteile wären gemeinfrei und könnten frei verwendet werden. Das würde einem großen Anteil von Urheberrechtsstreitigkeiten die Grundlage entziehen. Es wäre aber weiterhin nicht möglich, ganze Songs oder substantielle Songteile, wie Strophen und Refrains, in ein neues Werk zu übernehmen, wenn die ursprünglichen Werkteile eindeutig wiederkennbar bleiben.

Wenn man den Umstand der Rekombination von Melodiebausteinen zusammen mit der Tatsache sieht, dass auch Laien problemlos in der Lage sind, Popsongmelodien zu erfinden, man also der Argumentation folgt, dass Melodiekomposition im Wesentlichen musikalisches Handwerk ist, würde dies eine Abschaffung des starren Melodienschutzes nahelegen, was damit fast einer Abschaffung des Urheberrechts für Popsongs gleichkommen würde, denn die anderen Elemente, wie Akkordfolgen, Drumgrooves, Begleitfiguren, Sounds etc. sind bereits als solche nicht schutzwürdig. Die Frage wäre, was dann überhaupt noch geschützt ist? Es blieben weiter-

30 N.N., Court throws out musicologist, https://musicinfringement.art.blog/2020/04/ 24/court-throws-out-musicologist-judith-finells-expert-report-and-finds-no-infringe ment-by-raise-me-up-josh-groban/ (zuletzt abgerufen am 24.05.2020). 
hin künstlerisch sinnvolle Kombinationen aus an sich nicht schützbaren Elementen schützbar, was teilweise im Sinne eines "Gesamteindrucks" in der Rechtsprechung auch jetzt schon Anwendung findet ${ }^{31}$. Folgte man zudem dem Argument, dass Innovation und Originalität in der Popmusik vor allem in der "Interpretation“, also der konkreten Ausführung eines Songs zu finden ist, dann sollten zumindest konkrete Aufnahmen Urheberrechtsschutz genießen. Das würde eine interessante Verschiebung bedeuten, da das, was vorher nur unter das Leistungsschutzrecht fiel (einschließlich samplings aus Audioaufnahmen), nun genuin urheberrechtlich geschützt wäre.

Es ist aber klar, dass derlei Überlegungen nur den Charakter eines Gedankenexperiments haben, denn es steht nicht zu erwarten, dass sich in absehbarer Zukunft bedeutende Änderungen an der Formulierung des Gesetzestextes oder auch nur der Rechtspraxis der Kleinen Münze ergeben werden, dies käme einer Revolution gleich, an denen die Beteiligten kaum ein (finanzielles) Interesse haben könnten und zudem alle bisherigen Präzedenzfälle invalidieren würde. Aber jüngste Urheberechtsprozesse wie das „Blurred Lines“-Urteil ${ }^{32}$ oder der Fall von Katy Perry vs. Flame, haben neue Präzedenzen geschaffen haben, die aus der Sicht von vielen Künstlern und der Musikindustrie Anlass zu Sorge geben. So hat der Fall Katy Perry vs. Flame dazu geführt, dass Perry mit dem Argument der Trivialität und Vorbekanntheit der gegenständlichen Melodie verteidigt wurde, was aber im Wesentlichen ein Argument gegen die Kleine Münze darstellt. Dies könnte in der langfristigen Perspektive dazu führen, dass einige der hier aufgebrachten Argumente in die Diskussion miteinfließen. In der Tat hat sich im Fall von Katy Perry vs. Flame kurz vor Drucklegung eine überraschende Wendung ergeben, da die Richterin Christina A. Snyder das Urteil der Jury gegen Perry aufhob. ${ }^{33}$ Begründet wurde dies von Snyder damit, dass die streitgegenständlichen Melodien für sich genommen nicht hinreichend substantielle Originalität aufweisen würden, um den Prozess überhaupt zu rechtfertigen, was im Wesentlichen der hier dargelegten Argumentation folgt. Die Anwälte von Flame haben aber bereits angekündigt, gegen diese Entscheidung Berufung einzulegen.

31 LG München, Urt. v. 3.12.2008 - 21 O 23120/00, Still got the Blues.

32 US Court of Appeal for the Ninth Circuit, Urt. v. 21.3.2018 - Nr. 15-56880, Williams vs. Gaye.

33 Sisario., Katy Perry Prevails, https://www.nytimes.com/2020/03/17/arts/music/katy -perry-dark-horse.html (zuletzt abgerufen am 24.05.2020). 


\section{Fazit}

Wir haben in diesem Beitrag auf Grundlage neuerer musikwissenschaftlicher Erkenntnisse gezeigt, dass der starre Melodienschutz und die Kleine Münze sich weder mit der popmusikalischen Produktionspraxis noch mit den für Popmusik relevanten wahrnehmungspsychologischen Grundlagen noch mit statistischen Analysen von melodischen Bausteinen in Popmusikkorpora gut vereinbaren lassen. Zusätzlich haben wir versucht deutlich zu machen, dass es für die Erfindung von stilkonformen Popmelodien keines musikalischen Genies bedarf, sondern i. d. R. durch die Rekombination vorbestehender melodischer Bausteine erklärt werden kann. Zusammengenommen führt dies zum Argument, dass die Schaffung von Melodien de facto dem musikalischen Handwerk zuzuordnen ist. Dies würde dann nach gängiger Praxis aber sowohl die Kleine Münze als auch den starren Melodienschutz obsolet machen (oder den Melodienbegriff verschärfen bzw. einschränken). Zumindest sollte die Bestimmung, dass auch Teile aus fremden Werken nicht übernommen werden können, fallen gelassen oder stark eingeschränkt werden. Wenn die Schaffung von Melodien als persönliche geistige Schöpfung anzusehen ist, dann kann dies in unseren Augen nur für größere musikalische Strukturen gelten, was keine Änderungen der Gesetze, sondern nur der Rechtspraxis bedürfte.

Zusätzlich sei noch darauf verwiesen, dass auch Algorithmen der künstlichen Intelligenz teilweise jetzt schon in der Lage sind, hinreichend valide Melodien zu generieren ${ }^{34}$, auch wenn dies derzeit i. d. R noch menschlicher Auswahl und Nachbearbeitung bedarf. Das hat aber weitreichende Implikationen für das Urheberrecht, z. B., ob Computer-generierte Werke überhaupt schützbar sind, wer diesen Schutz erhält und ob man ein Computer-generiertes Werk der Urheberrechtsverletzung beschuldigen kann. Darüber hinaus unterminiert die Fähigkeit von Algorithmen zur Komposition von musikalischen Werken die Idee, dass ein Musikwerk immer eine persönliche geistige Schöpfung sein muss, dies ist dann nicht mehr - wie bisher - als selbstverständlich vorauszusetzen, was unseres Erachtens höhere Ansprüche an die zu fordernde Schöpfungshöhe menschlich geschaffener Musikwerke legen würde.

Schließlich sei noch angemerkt, dass bei einer Reform des musikalischen Urheberrechts die Funktion als Schutzinstrument von Künstlern er-

34 Colton et al., Beyond the Fence, in: Proceedings ICCC, o.S; Cope, Computer Models; Papadopoulos et al., Assisted Lead Sheet Compositions, in: Proceedings ICPPCP, o.S. 
halten bleiben muss, die in ihrer Arbeit unterstützt und angemessen entlohnt werden sollen. Die Frage steht aber im Raum, welche Leistungen von wem in der Popmusik wie geschützt werden sollen, so dass ein gerechter Ausgleich unter allen Beteiligten hergestellt werden kann und Popmusikkultur auch weiterhin auf einer professionellen und kommerziellen Basis existieren kann.

\section{Literatur}

Axis of Awesome. 4 Chords, https://www.youtube.com/watch?v=oOlDewpCfZQ (zuletzt abgerufen am 15.7.2020).

Baddeley, Alan, Working memory, Oxford 1986.

Bennet, Joe, Constraint, collaboration and creativity in popular songwriting teams. The act of musical composition: Studies in the creative process, in: D. Collins (Hrsg.), The act of musical composition: Studies in the creative process, Aldershot 2012, S. 139ff.

Bisges, Marcel, Die kleine Münze im Urheberrecht: Analyse des ökonomischen Aspekts des Werkbegriffs, Baden-Baden 2014.

Bregman, Albert S., Auditory Scene Analysis: The Perceptual Organization of Sound, Cambridge, Massachusetts 1990.

Cason, Robert J. S. / Müllensiefen, Daniel: Singing from the same sheet: Computational melodic similarity measurement and copyright law, International Review of Law, Computers \& Technology 26/1 2012, 25ff. doi:10.1080/13600869.2012.6 46786

Colton, S. / Llano, M. T. / Hepworth, R. / Charnley, J. / Gale, C. V. / Baron, A. / Pachet, F. / Roy, P. / Gervás, P. / Collins, N. / Sturm, B. / Weyde, T. / Wolff, D. / Lloyd, J., The Beyond the Fence Musical and Computer Says Show Documentary, in: Proceedings of the 7th International Conference on Computational Creativity. ICCC 2016, Paris 2016.

Cope, David. Computer Models of Musical Creativity. Cambridge, Massachusetts 2005.

de Laat, Kim: „Write a Word, Get a Third”: Managing Conflict and Rewards in Professional Songwriting Teams. Work and Occupations 42/2 (2015), $225 \mathrm{ff}$. doi:10.1177/0730888414562073

Döhl, Frédéric, Substantially Similar? Das Plagiat aus Sicht des Verhältnisses von Musik und Recht, in: Bung, Jochen / Gruber, Malte-Christian / Kühn, Sebastian (Hrsg.), Plagiate: Fälschungen, Imitate und andere Strategien aus zweiter Hand, Berlin 2011, S. 201ff. 
Freundlich, Kenneth. D. Brief of Amicus Curiae Musicologists in Support of Defendant's Renewed Motion For Judgement as a Matter of Law or Alternatively For a New Trial, https:/www.digitalmusicnews.com/wp-content/uploads/2020/01/ Katy_Perry_Flame_514_Amici_Brief_Filed.pdf (zuletzt abgerufen am 15.07.2020).

Frieler, Klaus, Rhythmus- und Tonhöhenmuster im europäischen Volkslied, Vortrag auf Arbeitstagung der Fachgruppe Systematische Musikwissenschaft. der Gesellschaft für Musikforschung, Köln, 2016.

Frieler, Klaus, Patternology. Melodic Pattern Usage in Charlie Parker's Solos, https://medias.ircam.fr/x54b408 (zuletzt abgerufen am 15.07.2020).

Frieler, Klaus / Müllensiefen, Daniel, Song and Harmonic Templates in Popular Music, in: Tillesen, P. / Prill, T. (Hrsg.), Whatever. Leipzig 2020, o.S.

Frieler, Klaus / Riedemann, Frank: Is Independent (Re)creation Likely to Happen in Pop Music? Musicae Scientiae 15/1 (2011), S. $17 \mathrm{ff}$.

Frith, S., Straw, W., \& Street, J. (Hrsg.). The Cambridge companion to pop and rock. Cambridge 2001.

Halpern, Andrea R. / Bartlett, J. C., Memory for melodies, in: Riess Jones, M. / Fay, R. R. / Popper, A. N. (Hrsg.), Music perception, Heidelberg 2010, S. $234 \mathrm{ff}$.

Hanser-Strecker, Carl-Peter, Das Plagiat in der Musik: Ein Beitrag zur Frage des urheberrechtlichen Schutzes von Werken der Musik, Frankfurt a. M. 1968.

Jörger, Thomas M., Das Plagiat in der Popularmusik, 1. Aufl., Baden-Baden 1992.

Kramarz, Volker, Warum Hits Hits werden: Erfolgsfaktoren der Popmusik: eine Untersuchung erfolgreicher Songs und exemplarischer Eigenproduktionen, Bielefeld 2014.

Miller, George A.: The magical number seven, plus minus two Some limits on our capacity for processing information, Psychological Review 63 (1956), S. 81ff.

Moore, Alan. F., Analyzing popular music, Cambridge 2003.

Müllensiefen, Daniel / Egermann, Hauke / Burrows, Sean, Radio Station Jingles: How statistical learning applies to a special genre of audio logos, in: Bronner, K. / Ringe, C. / Hirt, R. (Hrsg.), Audio Branding Yearbook 2014/2015, Baden-Baden 2015, S. $53 \mathrm{ff}$.

Müllensiefen, Daniel / Frieler, Klaus: Cognitive Adequacy in the Measurement of Melodic Similarity: Algorithmic vs. Human Judgments, Computing in Musicology 13 (2004), o.S.

Müllensiefen, Daniel / Pendzich, Marc: Court decisions on music plagiarism and the predictive value of similarity algorithms. Musicae Scientiae Discussion Forum 4B (2009), S. $257 \mathrm{ff}$.

Müllensiefen, Daniel / Wiggins, Geraint / Lewis, David, High-Level Feature Descriptors and Corpus-Based Musicology: Techniques for modelling Music Cognition, in: Schneider, A. (Hrsg.), Hamburger Jahrbuch für Musikwissenschaft: Vol. 25. Systematic and Comparative Musicology, Frankfurt a. M. 2008, S. $133 \mathrm{ff}$.

Norgaard, Martin: How Jazz Musicians Improvise: The Central Role of Auditory and Motor Patterns, Music Perception: An Interdisciplinary Journal, 31/3 (2014), S. 271ff. doi:10.1525/mp.2014.31.3.271. 
Owens, Thomas. Charlie Parker. Techniques of Improvisation. University of California 1974.

Papadopoulos, Alexandre / Roy, Pierre / Pachet, Francois, Assisted Lead Sheet Composition using FlowComposer, in: Proceedings of the 22nd International Conference on Principles and Practice of Constraint Programming. CP 2016, Toulouse, France 2016.

Pearce, Marcus T. / Wiggins, Geraint A.: Expectation in Melody: The Influence of Context and Learning, Music Perception 23/5 (2006), S.377ff. doi:10.1525/ mp.2006.23.5.377

Schmidt, Andre / Terhag, Jürgen, Songwriting: 40 Wege zum eigenen Song, 1. Aufl., Mainz 2010.

Seabrook, John, Songmachine: Inside the Hit Factory. New York City 2015.

Sisario, Ben, Katy Perry Prevails, https://www.nytimes.com/2020/03/17/arts/music/ katy-perry-dark-horse.html (zuletzt abgerufen am 24.05.2020).

Tversky, Amos / Kahneman, Daniel: Availability: A heuristic for judging frequency and probability, Cognitive Psychology, 5/2 (1973), S. 207ff. doi:10.1016/00100285(73)90033-9.

Zajonc, Robert B.: Attitudinal effects of mere exposure, Journal of Personality and Social Psychology 9/2, Pt. 2 (1968), S. 1ff. doi:10.1037/h0025848. 
